\title{
A STUDY OF MOBILITY MODELS FOR UAV COMMUNICATION NETWORKS
}

Haque Nawaz

Department of Computer Science, Shaheed Zulfikar Ali Bhutto Institute of Science and Technology, Karachi, and Sindh Madressatul Islam University, Karachi

(Pakistan)

E-mail: hnlashari@smiu.edu.pk

Husnain Mansoor Ali

Shaheed Zulfikar Ali Bhutto Institute of Science and Technology, Karachi (Pakistan)

E-mail: husnain.mansoor@szabist.edu.pk

Shafiq ur Rehman Massan

Mohammad Ali Jinnah University, Karachi (Pakistan)

E-mail:srmassan@hotmail.com

Recepción: 05/03/2019 Aceptación: 09/04/2019 Publicación: 17/05/2019

\section{Citación sugerida:}

Nawaz, H., Ali, H. M. y ur Rehman Massan, S. (2019). A Study of Mobility Models for UAV Communication Networks. 3C Tecnología. Glosas de innovación aplicadas a la pyme. Edición Especial, Mayo 2019, pp. 276-297. doi: http://dx.doi.org/10.17993/3ctecno.2019. specialissue2.276-297

\section{Suggested citation:}

Nawaz, H., Ali, H. M. \& ur Rehman Massan, S. (2019). A Study of Mobility Models for UAV Communication Networks. 3C Tecnología. Glosas de innovación aplicadas a la pyme. Special Issue, May 2019, pp. 276-297. doi: http://dx.doi.org/10.17993/3ctecno.2019. specialissue2.276-297 


\section{ABSTRACT}

The Unmanned aerial vehicle communication network (UAVCN) is a group or swarm of unmanned aerial vehicles which can be used for specific military and civilian applications without human intercession. This network faces the design problem which is based on network mobility. The frequent topology changes affect communication and collaboration among the UAVs (Unmanned aerial vehicles). To govern the movement pattern of UAVCN different mobility models needed to be studied in order to solve this communication issue. In this paper, mobility models are explored which provides the particular mobility pattern to resolve the problem of collaboration, communication and cooperation of UAVs. These models have been categorized into five groups and classified each group in detail. These mobility models provide the platform to understand and implement the unmanned aerial communication network for specific environment scenarios. The mobisim simulator tool is used to generate the mobility model s trajectories for different mobility models.

\section{KEYWORDS}

Mobility models, Unmanned aerial vehicles, Unmanned aerial vehicle communication networks, Wireless communication. 


\section{INTRODUCTION}

Recently the UAVCN is growing technology in the field of wireless communication and ad-hoc networking. The UAVs establish networking to communicate in the air which increases the demand to attain the larger area for a specific mission. Nowadays, it is an economic solution for different civilian and military monitoring and surveillance applications. These nodes mover freely in free space with a speed of 30 to $460 \mathrm{~km} / \mathrm{h}$. However, it creates problems in communication due to the high (Bujari, Calafate, Cano, Manzoni, Palazzi \& Ronzani, 2017). Eventually, this problem is not avoided due to sensitive and reliable real-time applications. However, researchers develop the new mobility models for a specific environment and applications to cope with the challenges of mobility. The routing is important in the communication of UAVs that depends upon the mobility models. These models of mobility cope with the speed deviations of UAVs. The random movement of nodes due to environment and location changes that needed to redefining the paths. However, the random mobility model is used to model UAVs communication scenarios. For the correlated movement of UAVs, the temporal-based model is used for critical situations or missions where the systematic movement of nodes needed. The group of UAVs which can fly in the space and collaborate with each other for a specific application. Figure.1 depicts the UAVCN network.

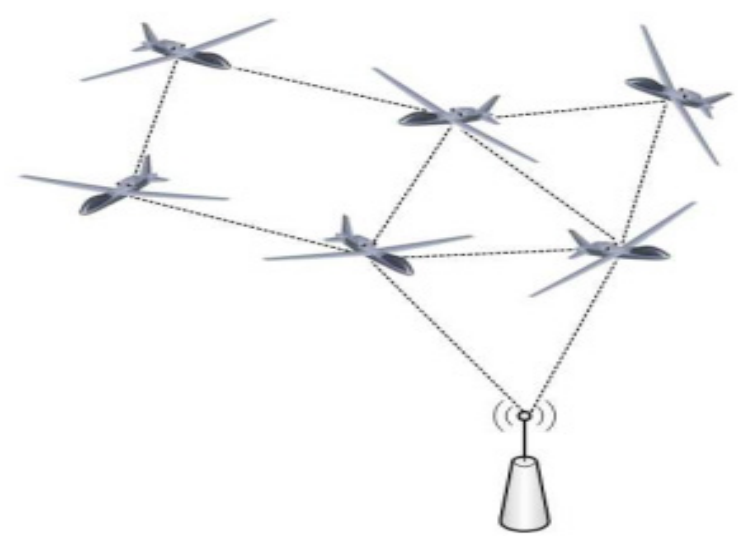

Figure 1. UAVCN Network. 
Generally, the mobility models have a greater role in the communication that provides the mobility patterns to generate pathways for UAVs communication. The novelty of this paper is to study the several mobility models suitable for Unmanned aerial vehicle communication networks for different situations and for different applications in this domain.

\section{LITERATURE REVIEW}

The UAVCN performance varies by mobility models and the type of applications. However, the movement patterns of UAVs are important for communication. The dynamic nature of these network nodes impacts on network features such as connectivity, distance, and density or capacity of the network. In the real world, dynamic and random patterns are different from actual patterns of movement (Yassein \& Damer, 2016). Therefore, the researchers explored this area by classifying the mobility models. In ad-hoc networking, the mobility models split into two classes. One of them is an entity and another is mobility models (Wang, Guan, Wang \& Wang, 2010). In this research, the authors explored the multi-hop routing and using different models evaluated and observed the significant impact on performance. It has studied that many authors explored existing mobile ad-hoc network mobility models, although the few have focused on UAVs ad-hoc mobility models. In Wan, Namuduri, Zhou and Fu (2013) authors proposed AN (AirBrone) network in which smooth turn mobility model has used which accounts for spatial and temporal dependency. The nodes trajectory could be predicted by speed and acceleration correlated information. In (Kuiper \& Nadjm-Tehrani, 2006) authors have discussed the scenarios of UAVs which having the memoryless pattern of mobility models. In the first scenario, the nodes have the memoryless movement and another scenario using the pattern of pheromones that help coordination among UAVs. In Ribeiro and Sofia (2011) authors discussed the Paparazzi mobility model for UAVs communication and compared with another random waypoint. Paparazzi mobility model has five movement patterns stayAt, waypoint, eight, oval and scan. In Bouachir, Abrassart, Garcia and Larrieu (2014) authors proposed Semi-Random Circular Movement mobi- 
lity model for UAV ad-hoc network. In Erim and Wright (2017), this paper, authors have examined RPGM (Reference Point Group Mobility model), GMMM (Gauss Markov mobility model), RWP (Radom Waypoint) and MGMM (Manhattan Grid mobility model) by using temporal dependent, spatial dependant, geographic-based and random based mobility model families. To create realistic ad-hoc network simulation environment these models are indispensable (Aschenbruck, Gerhards-Padilla \& Martini, 2008). For exploring of ad-hoc network protocol performance in mobile ad-hoc networking is varying significantly as compared to studying the unmanned aerial vehicle communication network. A survey carried out by authors for Airbrone Networks in which the mobility models are classified into two categories. One is traditional mobility models another is aerial ad-hoc network mobility models (Xie, Wan, Kim, Fu \& Namuduri, 2014).

\section{MOBILITY MODEL}

The UAVCN network mobility is contingent on two fundamental aspects, one of them is nodes location another is velocity change with respect to time (Yassein \& Damer, 2016). The study of node movement can be carried out by mathematical equations, mathematical modelling or simulation method. The simulation method is more reliable for mobility modelling and provides a better solution for complex problems. The mobility models which can be used for UAVCN are categorized as pure randomized mobility model, time dependant mobility model, path planned mobility mode, group mobility model and topology control based mobility model which are further classified and discussed as under.

\subsection{PURE RANDOMIZED MOBILITY MODELS}

The PRMM (Pure Randomized Mobility Models) are randomized in nature. UAVs are hovering in the environment randomly in terms of direction, movement time and speed (Kumari, et al., 2015). These models are commonly used to explore the area according to the mission by using UAVCN. The randomized models are simple and mostly used in research. The types of pure randomized 
models are RWP (Random Way Point) Mobility Model, RW (Random Walk) mobility model, RD (Random Direction) mobility model and MGM (Manhattan grid mobility model).

\subsubsection{RWP (RANDOM WAY POINT) MOBILITY MODEL}

The gap of time among the node direction and speed changes are coming under the umbrella of the RWP mobility model (Kumari, et al., 2015). Through this models UAV, s move in random positions in a particular region. This model supports the network nodes to move left, move right, and going straight. Figure 2. depict the RWP mobility model.

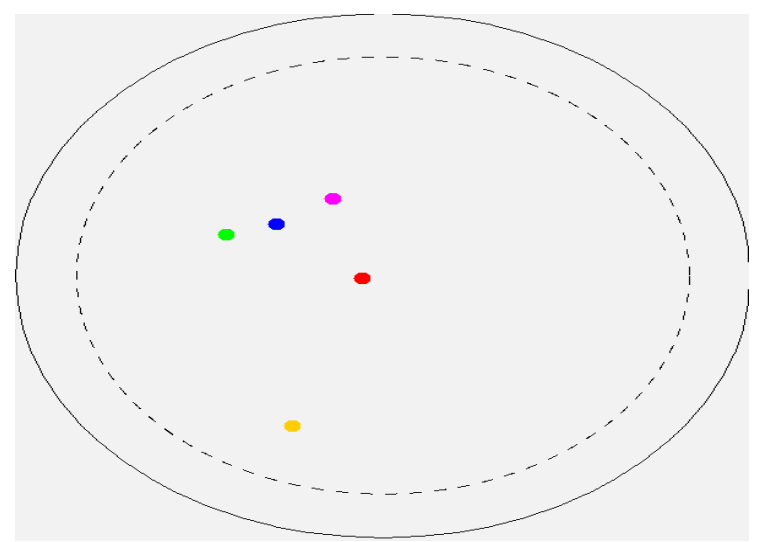

Figure 2. The random waypoint mobility model.

\subsubsection{RW (RANDOM WALK) MOBILITY MODEL}

The RW mobility model introduced for unpredictable random movement of numerous entities in the environment. It is based on the Brownian motion which mathematically described in 1926 by Einstein. In RW mobility model, the UAVs simulate this rough association preferring, each time, a random path. Every progress takes place in a constant time gap $t$ and travelled distance $d$, finally, at the ending, a new direction and velocity are calculated. However, a UAV jump the boundary of simulation region, the fresh direction is computed. It is known as memoryless mobility model because RW doesn't accumulate the information of its precedent position and movement. Figure 3 depict the RW mobility model. 


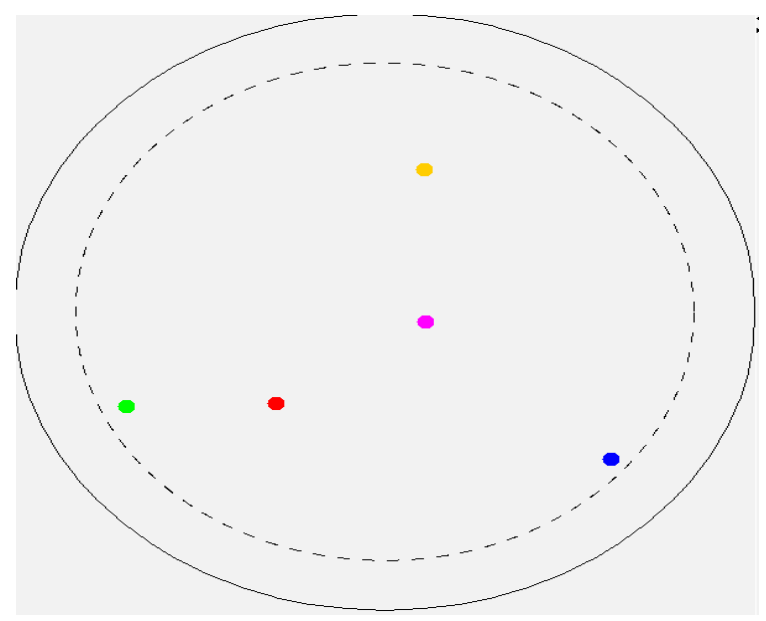

Figure 3. The random walk mobility model.

\subsubsection{RD (RANDOM DIRECTION) MOBILITY MODEL}

This mobility model was introduced to handle the problems of concentration of UAVs in the central part of the simulation region in the random waypoint mobility model because of the greater probability of movement towards the fresh destination close to the middle of simulation area. By random direction mobility model, every UAV choose a destination towards the edge of the simulation region. As the node reaches the edge, it yet again chooses an additional random destination position at the edge (Bujari, et al., 2017). Figure 4 depicts the RD mobility model.

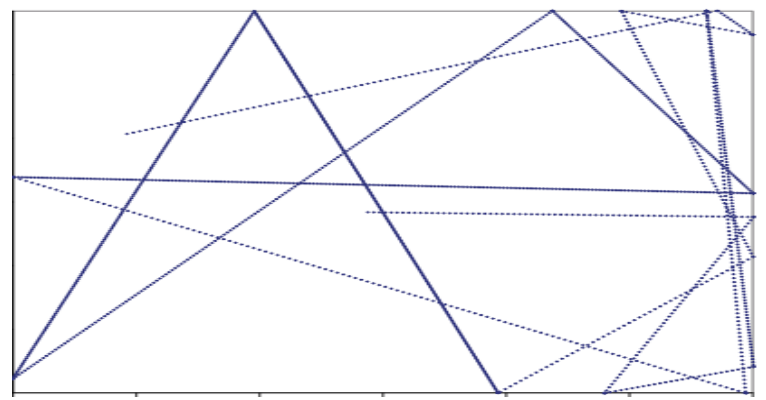

Figure 4. The random direction mobility model 


\subsubsection{MGM (MANHATTAN GRID MOBILITY MODEL)}

This model is used on the basis of the map-based approach. Where the UAVs movement takes place in the geographic boundaries. It indicates that the nodes travel in the predefined straight and perpendicular grids. The UAVs are permitted to move in the direction of north, south, east or west. At the defined grid the horizontal movement of node takes place with a probability of 0.5 and UAV can turn towards the corner with a probability of 0.25 (Wang, et al., 2010). This mobility model utilizes a grid path topology. GM mobility model was generally projected for the movement of the urban region, wherever the road design is extremely normal. This mobility model has also used an urban map for the movement of the straight and perpendicular direction. It utilizes a probabilistic approach for the choosing UAV movements, because, at every crossroads, a vehicle prefers whether to carry on moving in the similar path or to rotate. Figure 5 depicts the Manhattan grid mobility model.

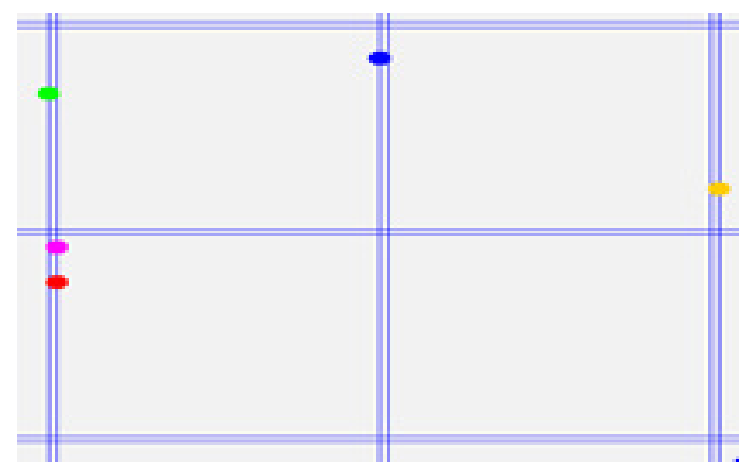

Figure 5. Manhattan grid mobility model.

\subsection{TDMM (TIME-DEPENDENT MOBILITY MODELS)}

The TDMM (Time-dependent mobility models) tries to avoid sharp changes in network nodes speed and direction. The smoothly changes in movement can be controlled by using different mathematical function. In these models UAVs depends upon the previous or earlier speed and direction (Guillen-Perez \& Cano, 2018). The types of time-dependent mobility models are BSA (Boundless simulation area) mobility model GMM (Gauss-Markov mobility model) (Regis, Bhunia \& Sengupta, 2016) and ST (Smooth turn) mobility model. 


\subsubsection{BSA (BOUNDLESS SIMULATION AREA) MOBILITY MODEL}

The BSA mobility model (Bujari, et al., 2017) employs an association among the earlier direction and speed and to the present one's conversation. It permits the UAV to move freely in the simulation region, do away with any edge effects on the simulation evaluation. Nevertheless, many simulation scenarios might discover unattractive the no-side effects that happen since the moving elsewhere of an edge and toward the inside from an additional one. Due to the teleportation effect, it would not fulfil the important conditions for the mission area of $2 \mathrm{D}$ applications.

\subsubsection{GMM (GAUSS-MARKOV MOBILITY) MODEL}

The GMM model based on the tuning constraint which contrasts the degree of unpredictability in the mobility or movement pattern. It is used for simulation of ad-hoc networks. This model is dissimilar with RWP due to simulation area is varied. In GMM (Gauss-Markov mobility) model, every UAV is primarily set to a particular speed and direction, later with respect to time, movement of UAV does randomly update the direction and the speed. Both parameters are measured based on the last position due to the fast movement (Bilal \& Khan, 2017). Figure 6 depicts the GMM model.

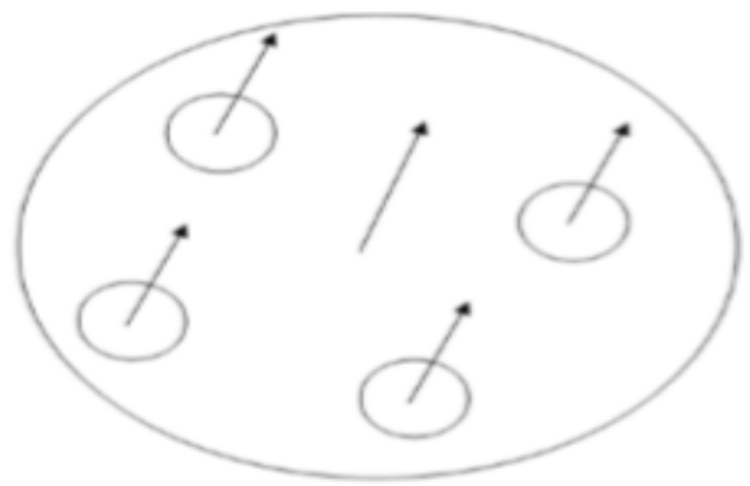

Figure 6. Gauss Markov mobility model. 


\subsubsection{ST (SMOOTH TURN) MOBILITY MODEL}

This mobility model (Wan, et al., 2013) permits the UAVs to travel in supple trajectories, comparing the increase of velocity rate of the UAVs across sequential and spatial coordinate. By means of this mobility model, every node selects a position in the space and then circle around it in anticipation of the node selects an additional turning point. The selected position should be vertical to the node direction, to make sure a horizontal trajectory. The period for the aeroplane to round in the region of the present circle position is formed to be exponentially distributed. Different conventional mobility models which strengthen vehicles to get ready quick turns, smooth turn practically capture the horizontal movement prototype of unmanned vehicles exclusive of insertion supplementary parameters. It has been studied that the cons of this model are lacking collision prevention method and boundary indication possessions due to the force of the moveable UAV on the region boundaries, which forces the UAV to unexpectedly amend its direction.

\subsection{PPMM (PATH PLANNED MOBILITY MODELS)}

The PPMM (Path planned mobility models) provides predefined path scheme to the network nodes. The UAVs follow that pattern and change the pattern at the end randomly. Similarly, change the pattern end to end in communication. The types of path planned mobility models are SRCM (Semi-random circular movement) mobility model and PPRZM (Paparazzi mobility model).

\subsubsection{SRCM (SEMI-RANDOM CIRCULAR MOVEMENT) MOBILITY MODEL}

SRCM mobility model is designed for the UAV's node which travels in a curving or bending manner. This model is employed for capturing of data and information through deploying UAVs in a specific area. Hence, every UAV is observing the specific area as permission. SCRM supports UAVs to communicate in a curved manner (Wang, et al., 2010). Figure 7 depict the SCRM mobility model. 


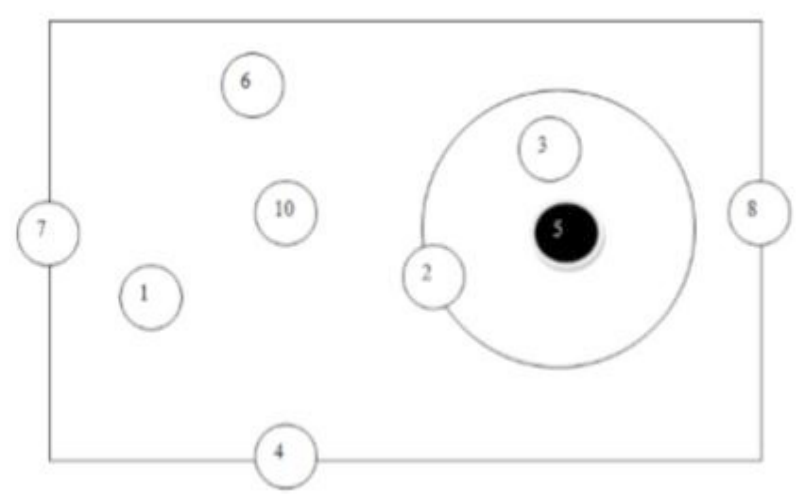

Figure 7. Semi-random circular movement mobility model.

\subsubsection{PPRZM (PAPARAZZI MOBILITY MODEL)}

The PPRZM is a stochastic flexibility exhibit that the copies paparazzi UAV conduct in light of the state machine. This model has 5 types of movements: one of them is staying at; this means over a fixed position UAV can hover, second is waypoint; In this movement UAV moves towards destination by using straight path, third is eight; In this movement UAV trajectory has the 8 shape from one place to another, scan; it scans the area by defining the two points through round trip, fifth is oval; it shifts round trip among 2 points and turn around when passing both points (Bouachir, Abrassart, Garcia \& Larrieu, 2014). Furthermore, this model can control a task since it accumulates utmost UAV imaginable progress by altering the opportunity of each growth sort as required. Figure 8 represents the paparazzi mobility model.

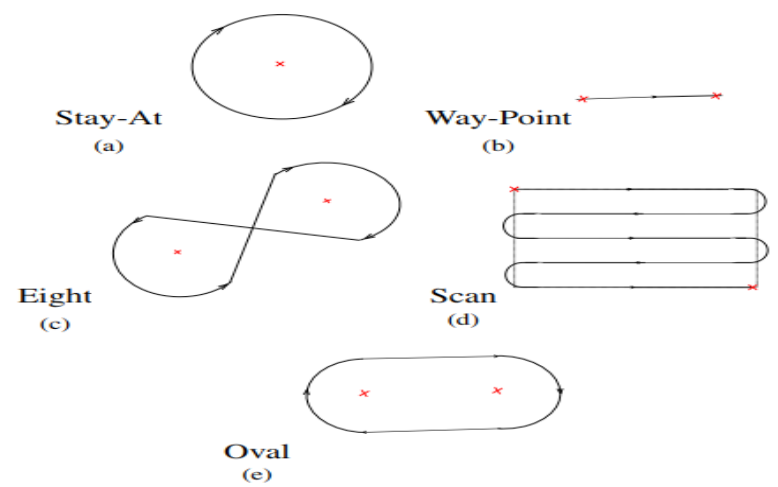

Figure 8. Paparazzi mobility model. 


\subsection{GMM (GROUP MOBILITY MODELS)}

The group mobility models are having spatial constraint among the UAVs. These nodes movement is controlled by a reference point. The UAVs randomly move around the reference point in a defined area. The GMM types are column mobility model and the nomadic community mobility model (Bujari, et al., 2017).

\subsubsection{CLMN (COLUMN MOBILITY MODEL)}

It was projected for inspecting or searching targeted application scenarios. Every UAV moves in the region of a reference position located on a specified line, which is moving in a frontward direction (Gupta, Sadawarti \& Verma, 2013). In specific, every UAV arbitrarily turns in the region of the reference position from beginning to end an entity mobility model, for example, an easy Random way. This model can avoid collision among UAVs, while every node moves in the region of a permanent position, individually these positions located distant commencing every one node. On the other hand, horizontal roll and speed modify are not there in this mobility model. Figure 9. represent the CLMN mobility model.

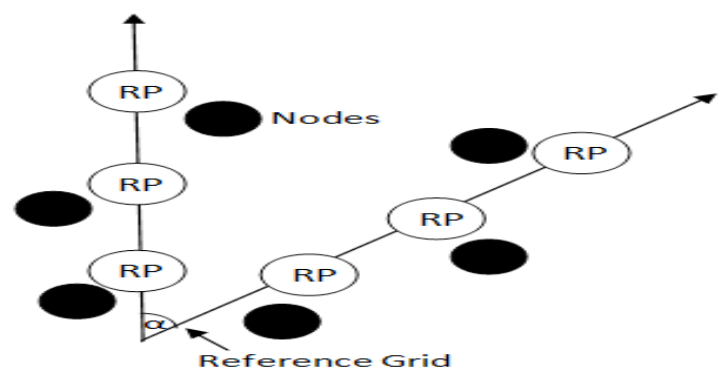

Figure 9. Column mobility model.

\subsubsection{NC (NOMADIC COMMUNITY) MOBILITY MODEL}

In this model UAVs moves arbitrarily in the region of a specified reference position, not including any constraint as compared to CLMN). The 5 nodes stirring around a mention position, it moves and turns in a definite direction. These nodes are able to move surrounded by rmax (maximum distance) as of the 
reference position. With respect to every time interval by using RWP (random waypoint), model reference position moves at a specific distance d. In Li, Zhang \& Li (2017), NC UAVs that split general spaces, generating collision events among the UAVs. Figure 10 depicts the NG mobility model.

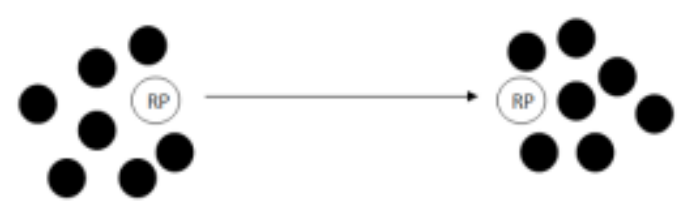

Figure 10. The nomadic community mobility model

\subsubsection{PRS (PURSUE) MOBILITY MODEL}

This mobility model is analogous to the Nomadic Community. In which UAVs endeavour to trail a specific target that is in a movement in a specific direction. The UAVs utilizes a simple random motion at the same time as trailing the target. For example, it performs the operation as similar to forces that try to catch the criminal by pursuing behind them (Singh \& Verma, 2014). Figure 11 depicts the PRS mobility model.

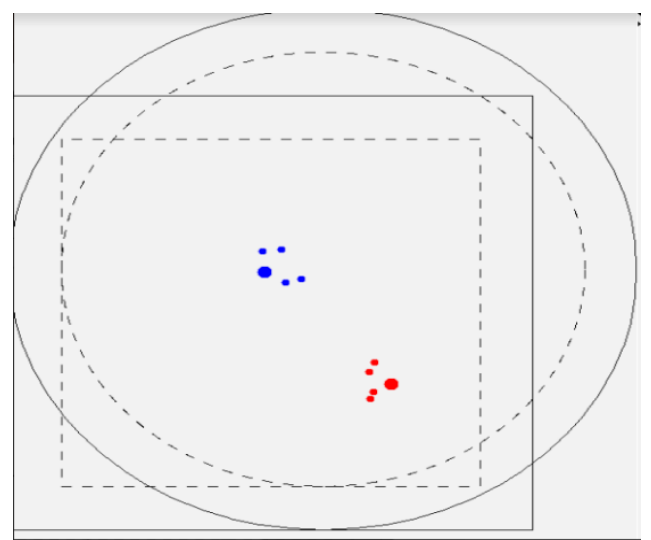

Figure 11. Pursue a mobility model. 


\subsection{TCMM (TOPOLOGY CONTROL MOBILITY MODEL)}

\subsubsection{PPR (DISTRIBUTED PHEROMONE REPEL) MOBILITY MODEL}

In Kuiper \& Nadjm-Tehrani (2006) DPR mobility model is projected, by means of to accomplish the objective of relating a vigorous and random movement mobile nodes that carry out an inspection to sense antagonistic earth targets. Every UAV preserve its individual pheromone map, it is a network of the region somewhere every cell enclose a time stamp on behalf of the previous time the cell was inspected. Just the once a UAV inspect the region, that marked on the map (Sanchez-Garcia, Garcia-Campos, Toral, Reina \& Barrero, 2015). This inspected region information is broadcasted to UAVs on a regular basis. In this model, UAV behaves like similar to the pheromone map. DPR is the enhanced version of the pheromone map.

\subsubsection{PHEROMONE-BASED MOBILITY MODEL}

Pheromone helps each UAV in the area of coverage for the development of UAV networks (Kuiper \& Nadjm-Tehrani, 2006). Every UAV highlights the zone and guide the other UAV in a specified area. In a sequence to increase the capacity, UAVs bring round in the direction of the expansion from beginning to end the zone with that does not find output till now. Similarly observed that the asymmetrical model is noticeably uncomplicated; nevertheless, it prompts ordinary results (Bouachir, et al., 2014). On the other hand, the pheromone base model has tremendously trustworthy scrutiny properties. This pheromone gives you an idea about, a pheromone guide is used to administer UAVs. Aerial Nodes trade data about their inspection region and turn right, left and proceed. Figure 12 represents the pheromone--based mobility model. 


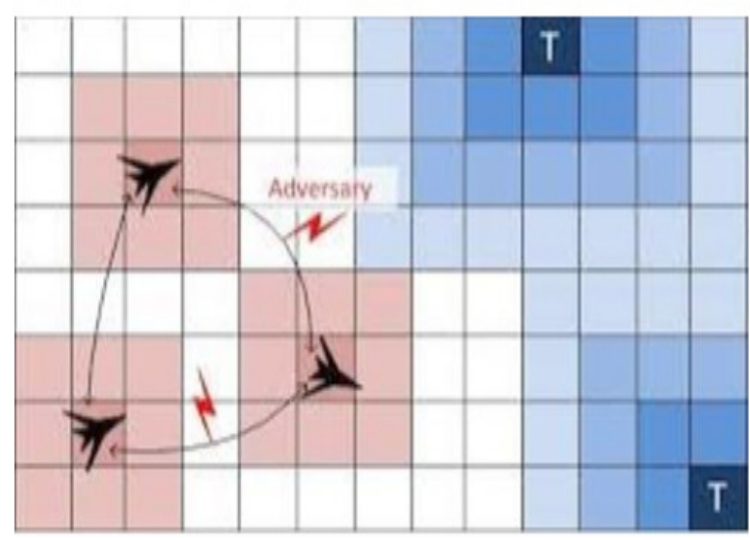

Figure 12. Pheromone-Based mobility model.

\subsubsection{MPB (MISSION PLAN-BASED) MOBILITY MODEL}

In MPB, flying idea information is predefined as well as the UAV's be capable to move further on by means of this plan (Kumari, et al., 2015). It entails that UAV's be in motion beside the strategic track every time, somewhere the UAV can arrive at the site of the mission and target area from where data is accessible. By using MPB when the mission is completed, the mobility records are generated and rationalized. Each UAV start and end drive are arbitrarily designated while flight time and the rate are mentioned. When a UAV touches destination before the completion of flight time, for continuous flying the UAV change the direction and starts new flight trip. Figure 13 depicts the MPB mobility model.

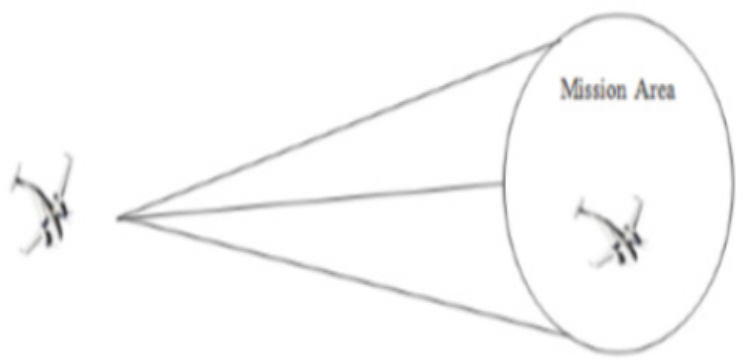

Figure 13. Mission-Based mobility model. 


\subsubsection{SDPC (SELF-DEPLOYABLE POINT COVERAGE) MOBILITY MODEL}

In Sanchez-Garcia, et al. (2015) this mobility model introduced for the scenarios of disaster. SDPC mobility model uses UAVs for a particular mission of disaster region, in order to construct a communication system that inspects the losses of the disaster which it happened. The objective of every UAV is to cover the maximum area. Figure 14 depicts the summary of mobility models.

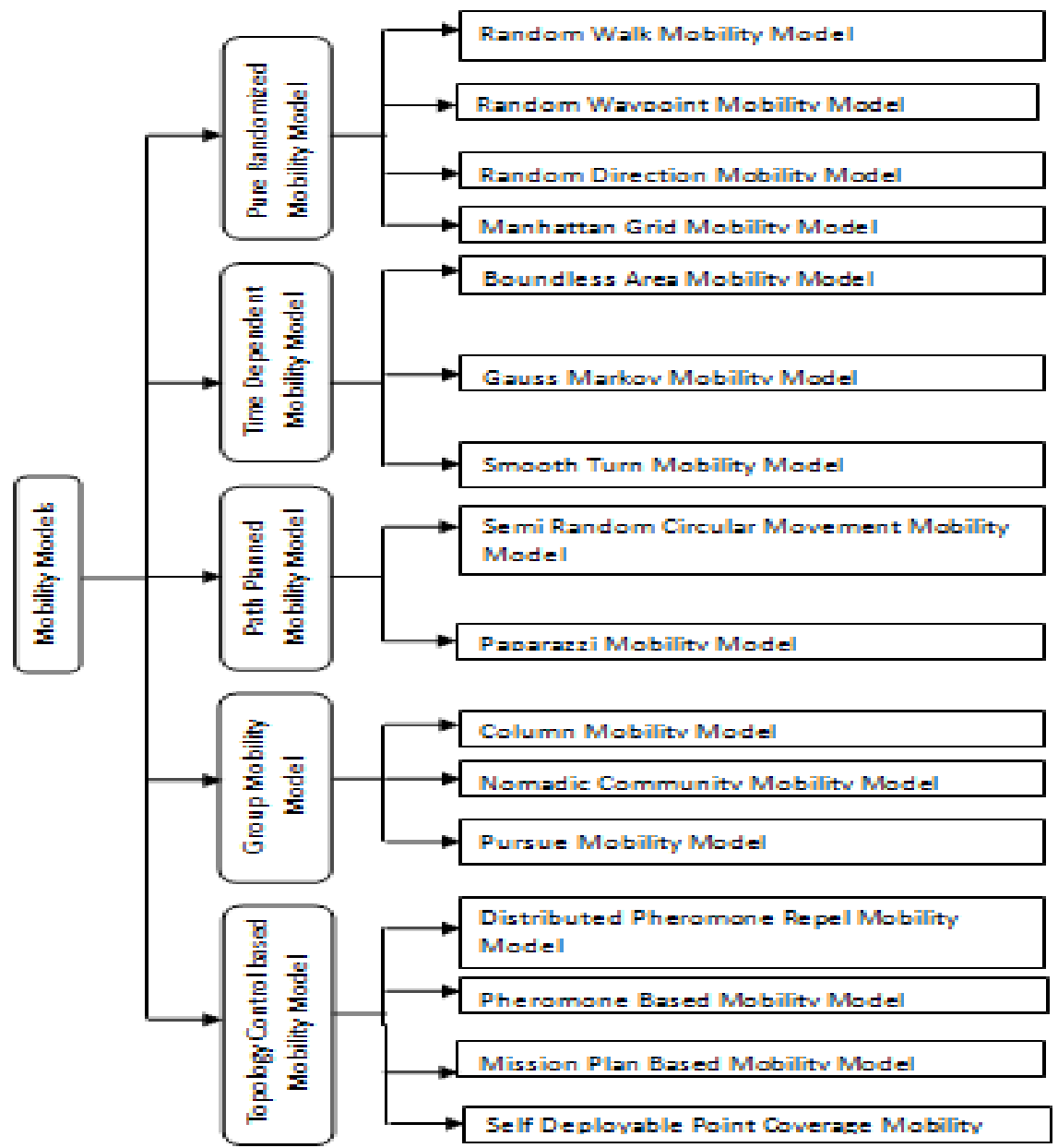

Figure 14. Summary of Mobility Models. 
Table 1. Categories of Mobility Models.

\begin{tabular}{|c|c|}
\hline Categories & Mobility pattern \\
\hline Pure randomized MM & RWMM \\
\hline & RWPMM \\
\hline Time dependant MM & RDMM \\
\hline & RGMM \\
\hline Path planned MM & BAMM \\
\hline & GMMM \\
\hline Group-based MM & STMM \\
\hline Topology control based MM & SRCMM \\
\hline & PMM \\
\hline & CMM \\
\hline
\end{tabular}

Table 1 Shows the five categories of mobility models and each category have different mobility patterns.

\section{CONCLUSION}

In this work, we have studied the state of the art related mobility models for specific environment scenarios trajectories in unmanned aerial vehicle communication network. The research community focused on the state of the art problem of a swarm of UAVs communication in this domain. However, in light of this, we have carried out the study which fulfil the current need of the research community in this domain. In this paper, we have explored the mobility models which provide the particular mobility pattern to resolve the problem of collaboration, communication and cooperation of UAVs. These models have been categorized into five groups and classified each group in detail. These mobility models provide the platform to understand and implement the unmanned aerial communication network for specific environment scenarios. The mobisim simulator tool we 
used to generate the mobility models trajectories for different mobility patterns. This facilitates the researchers to explore the emerging research area, ad hoc networking and UAV Communication Networks domain.

\section{ACKNOWLEDGEMENTS}

Authors of the article would like to acknowledge the unconditional and continued support, in terms of providing resources and encouraging environment, of SZABIST. Authors would also like to thank the anonymous reviewers for their valuable comments and suggestions which certainly helped in the improvement of the manuscript. Lastly, they are grateful to the IMCES-2019 for providing a platform for publishing the manuscript. 


\section{REFERENCES}

Aschenbruck, N., Gerhards-Padilla, E. \& Martini, P. (2008). A survey on mobility models for performance analysis in tactical mobile networks. Fournal of Telecommunications and Information Technology, 2, pp. 54-61.

Bilal, R. \& Khan, B. M. (2017). Analysis of Mobility Models and Routing Schemes for Flying Ad-Hoc Networks (FANETS). International fournal of Applied Engineering Research, 12(12), pp. 3263-3269.

Bouachir, O., Abrassart, A., Garcia, F. \& Larrieu, N. (2014). A mobility model for UAV ad hoc network. In 2014 international conference on unmanned aircraft systems (ICUAS) (pp. 383-388). IEEE.

Bujari, A., Calafate, C. T., Cano, J. G., Manzoni, P., Palazzi, C. E. \& Ronzani, D. (2017). Flying ad-hoc network application scenarios and mobility models. International Journal of Distributed Sensor Networks, 13(10). pp 1-17. doi: http://dx.doi.org/10.1177/1550147717738192

Bujari, A., Palazzi, G. E. \& Ronzani, D. (2017). FANET application scenarios and mobility models. In Proceedings of the 3rd Workshop on Micro Aerial Vehicle Networks, Systems, and Applications (pp. 43-46). ACM.

Erim, O. \& Wright, C. (2017). Optimized mobility models for disaster recovery using UAVs. In 2017 IEEE 28th Annual International Symposium on Personal, Indoor, and Mobile Radio Communications (PIMRC) (pp. 1-5). IEEE.

Gu, D. L., Pei, G., Ly, H., Gerla, M., Zhang, B. \& Hong, X. (2000). UAV aided intelligent routing for ad-hoc wireless network in single-area theater. In 2000 IEEE Wireless Communications and Networking Conference. Conference Record (Cat. No. 00TH8540) (Vol. 3, pp. 1220-1225). IEEE.

Guillen-Perez, A. \& Cano, M. D. (2018). Flying ad hoc networks: A new domain for network communications. Sensors, 18(10), p. 3571. doi: http:// dx.doi.org/10.3390/s18103571 
Gupta, A. K., Sadawarti, H. \& Verma, A. K. (2013). Performance analysis of MANET routing protocols in different mobility models. International fournal of Information Technology and Computer Science (IfITCS), 5(6), pp. 73-82.

Kuiper, E. \& Nadjm-Tehrani, S. (2006). Mobility models for UAV group reconnaissance applications. In 2006 International Conference on Wireless and Mobile Communications (ICWMC'06) (pp. 33-33). IEEE.

Kumari, K., Maakar, S. \& Sah, B. (2015). A brief survey of mobility model for FANET. In Proceedings of national conference on innovative trends in computer science engineering (pp. 106-108).

Kumari, K., Sah, B. \& Maakar, S. (2015). A survey: different mobility model for FANET. International Fournal of Advanced Research in Computer Science and Software Engineering, 5(6), pp. 1170-1173.

Li, X., Zhang, T. \& Li, J. (2017). A Particle Swarm Mobility Model for Flying Ad Hoc Networks. In GLOBECOM 2017-2017 IEEE Global Communications Conference (pp. 1-6). IEEE.

Regis, P. A., Bhunia, S. \& Sengupta, S. (2016). Implementation of 3d obstacle compliant mobility models for uav networks in ns-3. In Proceedings of the Workshop on $n s^{-3}$ (pp. 124-131). ACM.

Ribeiro, A. \& Sofia, R. (2011). A survey on mobility models for wireless networks, pp. 1-13.

Sanchez-Garcia, J., Garcia-Campos, J. M., Toral, S. L., Reina, D. G. \& Barrero, F. (2015). A self organising aerial ad hoc network mobility model for disaster scenarios. In 2015 International Conference on Developments of $E$-Systems Engineering (DeSE) (pp. 35-40). IEEE.

Singh, K. \& Verma, A. K. (2014). Applying OLSR routing in FANETs. In 2014 IEEE International Conference on Advanced Communications, Control and Computing Technologies (pp. 1212-1215). IEEE. 
Wan, Y., Namuduri, K., Zhou, Y. \& Fu, S. (2013). A smooth-turn mobility model for airborne networks. IEEE Transactions on Vehicular Technology, 62(7), pp. 3359-3370.

Wang, W., Guan, X., Wang, B. \& Wang, Y. (2010). A novel mobility model based on semi-random circular movement in mobile ad hoc networks. Information Sciences, $180(3)$, pp. 399-413.

Xie, J., Wan, Y., Kim, J. H., Fu, S. \& Namuduri, K. (2014). A survey and analysis of mobility models for airborne networks. IEEE Communications Surveys E Tutorials, 16(3), pp. 1221-1238

Yassein, M. B. \& Damer, N. A. (2016). Flying ad-hoc networks: Routing protocols, mobility models, issues. International fournal of Advanced Computer Science and Applications, 7(6), pp. 162-168. 